\title{
Unitary minimal Liouville gravity and Frobenius manifolds
}

\section{Belavin}

I.E. Tamm Department of Theoretical Physics, P.N. Lebedev Physical Institute, Leninsky ave. 53, 119991 Moscow, Russia

Department of Quantum Physics, Institute for Information Transmission Problems, Bolshoy Karetny per. 19, 127994 Moscow, Russia

E-mail: belavin@lpi.ru

ABSTRACT: We study unitary minimal models coupled to Liouville gravity using Douglas string equation. Our approach is based on the assumption that there exist an appropriate solution of the Douglas string equation and some special choice of the resonance transformation such that necessary selection rules of the minimal Liouville gravity are satisfied. We use the connection with the Frobenius manifold structure. We argue that the flat coordinates on the Frobenius manifold are the most appropriate choice for calculating correlation functions. We find the appropriate solution of the Douglas string equation and show that it has simple form in the flat coordinates. Important information is encoded in the structure constants of the Frobenius algebra. We derive these structure constants in the canonical coordinates and in the physically relevant domain in the flat coordinates. We find the leading terms of the resonance transformation and express the coefficients of the resonance transformation in terms of Jacobi polynomials.

Keywords: 2D Gravity, Conformal Field Models in String Theory, Matrix Models, Integrable Hierarchies

ArXiv EPRINT: $1405.4468 \mathrm{v} 2$ 


\section{Contents}

1 Introduction 1

2 Douglas approach and Frobenius manifolds 2

2.1 Frobenius algebra structure constants 4

2.2 Flat coordinates 6

3 Correlation functions $\quad 9$

$\begin{array}{lll}3.1 & \text { One-point functions } & 10\end{array}$

$\begin{array}{ll}3.2 & \text { Two-point functions } \\ \end{array}$

$\begin{array}{llr}4 \text { Conclusions } & 14\end{array}$

$\begin{array}{ll}\mathrm{A} C_{\alpha \beta \gamma} & 15\end{array}$

$\begin{array}{ll}\text { B Evaluation of } \frac{\partial \theta_{\lambda, k}}{\partial v_{\alpha}} & 16\end{array}$

\section{Introduction}

There are two independent approaches to 2D Liouville gravity [1]. The first method is based on the continual integral over Riemann metrics. After gauge fixing, it leads to the Liouville theory interacting with matter fields. If matter fields are represented by some minimal CFT model, then this theory is called minimal Liouville gravity (MLG). The main object of study in MLG is correlation functions, which are given by integrals over moduli of curves (with punctures) from differential forms constructed through conformal blocks [2]. Another approach to 2D gravity is based on the discrete approximation of 2D surfaces by graphs (studied in the double scaling limit). This approach is known as matrix models (MM) of 2D gravity [3-9].

One of the most important results concerning 2D gravity is the coincidence of the spectra of gravitational dimensions in the MLG and MM approaches. This observation led to the hypothesis that these two approaches are equivalent $[9,10]$. But the connection is not obvious, because the results for correlation functions do not coincide. As pointed out in [11], the problem in establishing the correspondence between MM and MLG is due to the freedom to add contact terms to the OPE. This effect leads to mixing the coupling constants. To identify the operators and the correlation functions, an explicit form of the resonance relations between couplings is needed. In [12], the relation between Liouville gravity and MM was studied for the Lee-Yang series of minimal models $(2,2 \mathrm{p}+1)$. The explicit form of the resonance relations in terms of Legendre polynomials was found. In [13], these results were generalized to $\left(3,3 p+p_{0}\right)$. It was shown that in this case, 
the basic requirements, i.e., absence of vacuum expectation values of physical operators, diagonal structure of the two-point correlators, and satisfaction of the conformal fusion rules, also leads to agreement between the two approaches on the level of the correlation functions.

The technique used in $[12,13]$ was to some extent specific to the particular cases under consideration. It was essentially based on the requirement that one of the parameters is small $(q=2,3)$. On the other hand, in [13], the connection of (p,q) critical points of MLG with the Frobenius manifold structure was significantly clarified. It was shown that the generating function of correlation numbers represents the logarithm of the tau-function of the integrable hierarchy related to some special Frobenious manifold. This connection can be used to obtain an explicit representation of the free energy in terms of the tau-function. The construction essentially requires knowing the structure constants of the Frobenius algebra. With these achievements, it is natural to tackle more general examples of MLG.

In this work, we use the Douglas approach and the abovementioned connection with the Frobenious manifold structure to study $(q+1, q)$ unitary models of MLG. The rest of the paper is organized as follows. In section 2, we briefly review the essence of the Douglas approach. We then introduce the Frobenious manifolds and find the solution of the Douglas string equation relevant for MLG. In this section, we also formulate the results for the structure constants of the Frobenius algebra. In section 3, we study conformal selection rules and the resonance relations. We apply the idea about the appropriate solution of the string equation formulated in the preceding section for one- and two-point correlation functions and discuss the resulting form of the resonance relations. Section 4 is for some concluding remarks.

\section{Douglas approach and Frobenius manifolds}

The MM of 2D gravity and the Douglas string equation approach [14] are widely studied in the literature (see, e.g., [11] and the references therein). Below, we briefly review the ideas relevant to our discussion.

The MM exhibits an infinite set of multi-critical points. In the scaling limit near the $(p, q)$ critical point, the singular part of the free energy $\mathcal{F}$ of the $p$-matrix model is constructed in terms of the solution of the string equation

$$
[\hat{P}, \hat{Q}]=1,
$$

where $\hat{P}$ and $\hat{Q}$ are two differential operators,

$$
\begin{aligned}
& \hat{Q}=\left(\frac{d}{d x}\right)^{q}+\sum_{\alpha=1}^{q-1} u_{\alpha}(x)\left(\frac{d}{d x}\right)^{q-\alpha-1}, \\
& \hat{P}=\frac{p+q}{q^{2}} \hat{Q}_{+}^{\frac{p}{q}}+\sum_{\alpha=1}^{q-1} \sum_{k=1} \frac{k q+\alpha}{q^{2}} t_{k, \alpha} \hat{Q}_{+}^{k+\frac{\alpha}{q}-1},
\end{aligned}
$$

and the plus sign means that only nonnegative powers of $d / d x$ are taken in the series expansion of the pseudo-differential operator. The constants $t_{k, \alpha}$ are usually called "times". This choice of the basis in the parameter space is called the KdV frame. 
The solution $u_{\alpha}^{*}(x)$ of the string equation (2.1) is related to the nonsingular part of the free energy

$$
\frac{\partial^{2} \mathcal{F}}{\partial x^{2}}=u_{1}^{*}(x)
$$

The main conjecture about the connection between the Matrix Models of 2D gravity and the continuous Liouville approach is that for the appropriate solution of the string equation the singular part of the free energy $\mathcal{F}$ coincides with the generating function of correlators of different topologies in the $(p, q) \mathrm{MLG} .{ }^{1}$ One further point is worthy of note. It may seem from the above simple definition that this construction comprises exhaustive information about the theory and that the remaining questions are quite technical and straightforward. But the problem is that if the partition function is the subject of the Douglas string equation, then there still remains much ambiguity related to the choice of the point in the space of theories (the point in the parameter space) and the choice of the appropriate coordinates in this space such that this particular setup leads to correct answers for the correlation functions. The transformation from the initial coordinates $t_{k, \alpha}$ to the appropriate "Liouville" coordinates $\lambda_{m n}$ is known as a resonance transformation [11] (it is discussed in detail below). Hence, there is a conceptual question of clarifying the meaning of these choices as well as the meaning of the other possible solutions of (2.1) and the relations between them.

In what follows we restrict our attention to the planar limit corresponding to the spherical topology. In this case, we are dealing with the dispersionless limit of the string equation when $d / d x$ is replaced with the variable $y$ and the commutator is replaced with the Poisson bracket. In particular, the operator $\hat{Q}$ transforms to the polynomial

$$
Q(y)=y^{q}+u_{1} y^{q-2}+u_{2} y^{q-3}+\cdots+u_{q-1} .
$$

In the planar limit, we are left with the system of first-order PDEs and the Douglas string equation takes the simple form $[13,15]$

$$
\frac{\partial S}{\partial u_{k}}=0
$$

where the so-called action is

$$
S\left[u_{\alpha}(x)\right]=-\operatorname{res}_{y=\infty}\left(Q^{\frac{p+q}{q}}+\sum_{n, m} t_{m n}(\mu, \lambda) Q^{\frac{p m-q n}{q}}\right),
$$

where $n, m$ numerate the primary fields in the underling minimal model. In this paper we will concentrate on the most important class of MLG theories related to the unitary minimal models $M(q+1, q),(p=q+1)$ coupled to Liouville gravity. Technically, because of the properties of the spectrum of the gravitational dimensions in the unitary models, this specification fixes the special form of the resonance transformation which will be discussed below and, hence, the form of the action (2.7). In particular, for the unitary minimal models the sum in (2.7) is fixed to be $1 \leq n \leq m \leq q-1$. On the other hand, as it

\footnotetext{
${ }^{1}$ The generating function can be thought of as a partition function of the perturbed minimal model coupled to Liouville gravity, which is why the generating function is called sometimes the partition function.
} 
was already seen in the $M(p, 3)$ case [13], i.e., for $q=3$, the analysis for general $p$ is mainly reduced to the consideration of different but analogous cases. The classification of these cases depends on the relation between the two coprime integers $p, q$, namely, on the quotient and the remainder produced after dividing $p$ by $q$. Meanwhile, in what follows, when the consideration is applicable for general $M(p, q)$ we leave the parameter $p$ unfixed.

The singular part of the partition function is expressed [13] in terms of (2.7) as an integrated (closed) one-form

$$
\mathcal{F}=\frac{1}{2} \int_{0}^{\mathbf{u}^{*}} \widetilde{C}_{k}^{i j} \frac{\partial S}{\partial u^{i}} \frac{\partial S}{\partial u^{j}} d u^{k}
$$

where $\mathbf{u}^{*}$ is a suitable solution of the string equation (2.6) and $\widetilde{C}_{k}^{i j}$ are the structure constants $^{2}$ of the Frobenius algebra discussed in the next section. Hence, according to our basic idea, this function gives the generating function of $(p, q)$ MLG

$$
\mathcal{F}=\left\langle\exp \sum_{m, n} \lambda_{m, n} O_{m, n}\right\rangle,
$$

where the Liouville coupling constants $\lambda_{m, n}$ are expressed in terms of the times $t_{m n}$ via the resonance transformation. Using the Douglas approach to solve MLG thus requires three main ingredients: the appropriate solution of the string equation, the resonance relations between times in the KdV frame and Liouville couplings, and, finally, the structure constants of the Frobenius algebra.

\subsection{Frobenius algebra structure constants}

In general, a Frobenius algebra $A$ is a commutative algebra with unity equipped with a nondegenerate invariant pairing $A \otimes A \rightarrow \mathbb{C}$. The invariance means that for any three vectors $a, b, c$ in $A$ :

$$
(a \cdot b, c)=(a, b \cdot c) .
$$

The Frobenius manifold $M[16]$ is defined as a Riemannian manifold with the flat metric $g_{\mathbf{v}}$ compatible with the additional structure of the Frobenius algebra $A$ associated with each point $\mathbf{v}$ (or with each tangent space $T_{\mathbf{v}} M$ ). Let $v^{1}, \ldots, v^{n}$ be the flat coordinates on an $n$-dimensional Frobenius manifold $M$. The algebra $A$ is identified with the tangent space $T_{\mathbf{v}} M$ by

$$
e_{\alpha} \leftrightarrow \frac{\partial}{\partial v^{\alpha}}
$$

where $\alpha$ runs from 1 to $n$. We hence have the following multiplication rule for the tangent vectors at any point $\mathbf{v} \in M$ :

$$
\frac{\partial}{\partial v^{\alpha}} \cdot \frac{\partial}{\partial v^{\beta}}=c_{\alpha \beta}^{\gamma}(\mathbf{v}) \frac{\partial}{\partial v^{\gamma}}
$$

The axiom

$$
\frac{\partial}{\partial v^{\delta}} c_{\alpha \beta \gamma}(\mathbf{v})=\frac{\partial}{\partial v^{\gamma}} c_{\alpha \beta \delta}(\mathbf{v})
$$

\footnotetext{
${ }^{2}$ We reserve $\mathrm{C}$ without the tilde for the flat coordinate system.
} 
defines the structure of the Frobenius manifold (see, e.g., [13] for more details about properties of Frobenius manifolds).

In Liouville gravity, we deal with a particular example of the Frobenius manifold where the corresponding algebra is defined as an algebra of polynomials $C[y]$ modulo the polynomial $Q^{\prime}=d Q / d y$, where $Q$ is defined in (2.5). The parameters $u_{i}$ in (2.5) are the coordinates on the Frobenius manifold. We call these coordinated canonical coordinates. In the basis $e_{1}, \ldots, e_{q-1}$, where $e_{k}=y^{q-1-k}$, the algebra looks like

$$
e_{i} e_{j}=\widetilde{C}_{i j}^{k} e_{k} \bmod Q^{\prime}
$$

and the metric $g_{i j}$ is given by

$$
g_{i j}:=\underset{y=\infty}{\operatorname{res}} \frac{e_{i} e_{j}}{Q^{\prime}} .
$$

The structure constants in (2.14) are exactly those that appeared in (2.8). The metric $g_{i j}$ (and its inverse) is used to raise and lower the indices. Using this, we find

$$
\widetilde{C}_{i j k}=\operatorname{res}_{y=\infty} \frac{e_{i} e_{j} e_{k}}{Q^{\prime}} .
$$

In particular, the metric itself is expressed in terms of the structure constants $g_{i j}=\widetilde{C}_{i j q-1}$. In each particular case, i.e., for a given $q$, the structure constants can be directly evaluated from the definition (2.16). For example, for $q=5$, we find

\begin{tabular}{|cccc|cccc|cccc|cccc|}
$\widetilde{C}_{1}^{i j}$ & & & & $\widetilde{C}_{2}^{i j}$ & & & $\widetilde{C}_{3}^{i j}$ & & & $\widetilde{C}_{4}^{i j}$ & & \\
\hline 5 & 0 & 0 & 0 & 0 & 5 & 0 & 0 & 0 & 0 & 5 & 0 & 0 & 0 & 0 & 5 \\
0 & $-3 u_{1}$ & $-2 u_{2}$ & $-u_{3}$ & 5 & 0 & 0 & 0 & 0 & 5 & 0 & 0 & 0 & 0 & 5 & 0 \\
0 & $-2 u_{2}$ & $-u_{3}$ & 0 & 0 & 0 & $-2 u_{2}$ & $-u_{3}$ & 5 & 0 & $3 u_{1}$ & 0 & 0 & 5 & 0 & $3 u_{1}$ \\
0 & $-u_{3}$ & 0 & 0 & 0 & 0 & $-u_{3}$ & 0 & 0 & 0 & 0 & $-u_{3}$ & 5 & 0 & $3 u_{1}$ & $2 u_{2}$
\end{tabular}.

It is worth noting that such a simple linear dependence characterizes the structure constants in $u$ coordinates only if two indices are raised. For all indices down, the dependence is nonlinear. This example demonstrates the following properties of the structure constants $\widetilde{C}_{k}^{i j}$. For a given index $k$, it has a block diagonal structure, and regarded as a function of $i$ and $j$, it depends on the combination $i+j$, which means that its counter-diagonal elements are the same. These properties together with the explicit calculations for particular values of the parameter $q$ (one of which is shown above) suggest the general form of the structure constant in $\mathbf{u}$ coordinates:

$$
\widetilde{C}_{k}^{i j}=(q+k-i-j+1) u_{i+j-k-2} \widetilde{\Theta}(i, j, k),
$$

where $\widetilde{\Theta}(i, j, k)$ is given by

$$
\widetilde{\Theta}(i, j, k)=\left\{\begin{aligned}
1 & \text { if } i, j \leq k \quad \text { and } i+j>k \\
-1 & \text { if } i, j>k \text { and } i+j \leq k+q \\
0 & \text { otherwise. }
\end{aligned}\right.
$$

In (2.17), we imply that $u_{-1}=1, u_{0}=0$. So far, we have not found a proof of the result (2.17). It therefore remains a conjecture verified up to $q=15$. 
The result (2.17) allows writing the partition function (2.8) in the form

$$
\mathcal{F}=\frac{1}{2} \int_{0}^{\mathbf{u}^{*}} \widetilde{\Omega}_{i} d u^{i}
$$

where

$$
\widetilde{\Omega}_{i}=q \sum_{k, j}^{k+j=i+1} \frac{\partial S}{\partial u_{k}} \frac{\partial S}{\partial u_{j}}+\left[\sum_{l \geq i+3}^{2 i} \sum_{k, j \leq i}^{k+j=l}-\sum_{l \geq 2 i+2}^{i+q} \sum_{k, j>i}^{k+j=l}\right](q+i-l+1) u_{l-i-2} \frac{\partial S}{\partial u_{k}} \frac{\partial S}{\partial u_{j}} .
$$

Hence, the found structure constants (2.17) together with the explicit form of the resonance relations, if it were known, could lead to a rather simple expression for the generating function of correlators. But one piece of data is missing: which solution of the string equation is related to minimal models coupled to gravity? We discuss this question in the next section.

\section{$2.2 \quad$ Flat coordinates}

It was claimed in [17] that only one of the solutions of the string equation is physical, i.e., it leads to zero one-point correlators. The proof of this statement is based on the counting of the free parameters (the parameters of the resonance transformation in our language) and the numbers of the constraints which arise from the requirement that for the order parameters (the fields with $m=n$ ) the one-point correlation functions vanish. It can be shown that all the parameters and hence the solution of the string equation are fixed uniquely by these constraints (for more details see [17]).

Hence, we just have to find the correct solution. In the $q=2$ case, there is only one solution (modulo some physically irrelevant phase factors). For $q=3$, when there are two coordinates $u_{1}$ and $u_{2}$, the situation is more subtle because the string equations have now a number of essentially different solutions. It was found in [13] that there exists only one particular solution with the necessary properties. The correctness of the choice was verified by computing the correlators. As a bonus, it turned out that the integration contour can be taken along one of two axes because $u_{2}^{*}=0$ (for all couplings equal to zero) for this particular solution. We recall that the integral is independent of the contour because the integrand is a closed one-form.

For $q>3$, the situation becomes more complicated. The computations show that unlike the $q=2,3$ cases, the roots of the string equation with only one nonzero component $^{3}$ are nonphysical in the sense that using these roots in the expression (2.19) for the partition function, we cannot satisfy one of the basic requirements that one-point functions of physical fields are equal to zero. The consideration in the canonical coordinates now becomes more involved because the contour of the integration does not go along one of the axes. In brief, we conclude that the appropriate physical solution of the Douglas string equation corresponding to the general $(p, q)$ MLG for an arbitrary value of the parameter $q$ seems complicated in the canonical coordinates $u_{i}$.

\footnotetext{
${ }^{3}$ Here the couplings equal to zero.
} 
Our idea is to find a transformation from the canonical coordinates to some new coordinates such that the physical solution would have the simplest form in the new coordinate system. We find that there exist coordinates $v_{i}$ such that the physical solution in the zeroth order of the expansion in the coupling constants becomes $\left(v_{1}, 0,0, \ldots\right)$, i.e., $v_{k>1}=0$, similarly to what happens in the canonical coordinates for $q=2,3$. Our calculations for different values of the parameter $q$ led to the conclusion that the correct choice of the coordinates coincides with the choice of the flat coordinates on the Frobenius manifold. The explicit form of the transformation from the canonical to the flat coordinates is given by the equality

$$
y=Q^{\frac{1}{q}}(y)-\frac{1}{q}\left(\frac{v_{1}}{Q^{\frac{1}{q}}(y)}+\frac{v_{2}}{Q^{\frac{2}{q}}(y)}+\cdots+\frac{v_{q-1}}{Q^{\frac{q-1}{q}}(y)}\right)+\mathcal{O}\left(\frac{1}{Q^{\frac{q+1}{q}}(y)}\right),
$$

where $Q(y)$ is defined in (2.5).

The flat coordinates have a number of useful properties. First, the metric in these coordinates is constant. It has the simple form

$$
\eta_{\alpha \beta}=\delta_{\alpha+\beta, q}
$$

The flat coordinates are expressed explicitly in terms of $u_{i}$ :

$$
v_{i}=\theta_{i, 0}
$$

Here, $\theta_{i, k}(k \in \mathbb{N})$ is an important object of the Frobenius manifold structure which is relevant for the existence of infinite number of commuting flows and for the relation with the integrable hierarchies. (It represents a one-parameter family of deformed flat connections on the Frobenius manifold; see, e.g., [13] for more details). It is given by

$$
\theta_{\alpha, k}=-c_{\alpha, k} \underset{y=\infty}{\operatorname{res}} Q^{k+\frac{\alpha}{q}}(y)
$$

with

$$
c_{\alpha, k}^{-1}=\left(\frac{\alpha}{q}\right)_{k+1},
$$

where $(a)_{n}=\Gamma(a+n) / \Gamma(a)$ is the Pochhammer symbol. The explicit form of the polynomial $Q$, evaluated in the flat coordinates on the solution of the string equation for the couplings equal to zero, is

$$
\left.Q(y)\right|_{\vec{v}_{0}}=y^{q}+q \sum_{k}^{2 k-1<q}\left(\begin{array}{c}
q-k-1 \\
k-1
\end{array}\right)\left(\frac{v_{1}}{q}\right)^{k} y^{q-2 k} .
$$

This expression is exactly the one that appeared in the planar limit of the $Q$ operator considered in [17]. The connection with the flat coordinates clarifies the geometric meaning of this choice of the $Q$ operator.

Let us emphasize at this point that by considering examples for different values of the parameter $q,(q=3,4,5)$, we have found that in the canonical coordinates, for each particular case, we can find some special solution of the string equation such that for this solution 
we can construct the resonance transformation so that the one-point correlation functions of all operators except unity operator are zero. We call it physical ${ }^{4}$ solution. Analysing these examples we have found that there exist some coordinate transformation, such that in the new coordinates this solution takes simple form $v_{\alpha>1}=0$. One simple motivation to search such transformation is the form of the partition function (2.8), since in the new coordinates we have no any problem with the choice of the integration contour. Then we have found out that the coordinate transformation in each particular case coincides with the transformation from the canonical coordinates to the flat coordinates on the Frobenius manifold (2.21). At the moment we conjecture that this picture persists for arbitrary value of the parameter $q$. Using the properties of the flat coordinates and analysing the form of the action $S$ (in particular the zeroth order coefficient $S^{(0)}$ ), we will see in the next section that this physical solution does exist for arbitrary values of the parameter $q$.

Hence, we find that the string equation has one particular solution, denoted by $\mathbf{v}^{*}$ in what follows, that is equal to $\left\{v_{1}^{*}, 0,0, \ldots\right\}$ in the zeroth order in coupling constants. It is shown in the next section that for this solution, the one-point correlation functions are zero and the two-point functions satisfy the diagonality requirement.

The structure constants in the flat coordinates are given by

$$
C_{\alpha \beta \gamma}=-q \underset{y=\infty}{\operatorname{res}} \frac{\frac{\partial Q(y)}{\partial v^{\alpha}} \frac{\partial Q(y)}{\partial v^{\beta}} \frac{\partial Q(y)}{\partial v^{\gamma}}}{Q^{\prime}(y)} .
$$

In particular, because $C_{1 \alpha \beta}=\eta_{\alpha \beta}$, we obtain

$$
C_{1}^{\alpha \beta}=\delta_{\alpha+\beta, q} \quad \text { and } \quad C_{\alpha}^{q-1, \beta}=\delta_{\alpha, \beta} .
$$

Finally, we note that because of the properties of the flat metric $\eta_{\alpha \beta}$, we obtain the useful relation

$$
v^{\alpha}=v_{q-\alpha} .
$$

Although we have obtained explicit structure constants in the canonical coordinates, we have not yet found the general answer for the structure constants in the flat coordinates. Nevertheless, taking the form of the generating function into account, we can note that to analyse one- and two-point correlation functions, we must know the structure constants in the flat coordinates only on the line $v_{k>1}=0$. This becomes clear when we take into account that the contour independence of the expression (3.1) can be used to choose it along $v_{1}$ and also that for one- and two-point correlation functions, the derivatives act only on the integrand.

To find the structure constant, we perform the coordinate transformation with the expression $(2.17)^{5}$

$$
C_{\alpha \beta \gamma}=\frac{\partial u^{i}}{\partial v^{\alpha}} \frac{\partial u^{j}}{\partial v^{\beta}} \frac{\partial u^{k}}{\partial v^{\gamma}} \widetilde{C}_{i j k}
$$

\footnotetext{
${ }^{4}$ To prove that this solution indeed corresponds to the minimal Liouville gravity we have to verify that all n-point correlators satisfy the necessary selection rules. In this paper we perform the check for one- and two-point correlators.

${ }^{5}$ We use Greek and Latin alphabets for the respective $\mathbf{v}$ and $\mathbf{u}$ coordinate systems.
} 
This allows obtaining an explicit form of the expansion in the vicinity of $\mathbf{v}^{*}$. We note that in the $n$-point correlation function, the contribution gives a maximum $(n-3)$ terms in this expansion. The explicit results for the first terms is presented below. To represent the answer compactly, we introduce the function $\Theta_{A, B}(x)$ such that $\Theta_{A, B}(x)=1$ if $x \in[A, B]$ and is zero otherwise. The result for the structure constant in the flat coordinates on the line $v_{i>0}=0$ is

$$
C_{\alpha \beta \gamma}=\Theta_{1, q-1}(\alpha+\beta-\gamma)\left(-\frac{v_{1}}{q}\right)^{\frac{\alpha+\beta+\gamma-q-1}{2}} \text { if } \frac{\alpha+\beta+\gamma-q-1}{2} \in \mathbb{N} \text {, otherwise } 0 .
$$

In (2.31), we assume the ordering $\alpha \geq \beta \geq \gamma$. Because this tensor is symmetric, this information gives the complete answer. We give some details of the derivation in appendix A.

\section{Correlation functions}

Our aim in this section is to determine the correlation functions in MLG using the special properties of the flat coordinates. All required information concerning these correlators is encoded in the partition function. In the flat variables,

$$
\mathcal{F}=\frac{1}{2} \int_{0}^{\mathbf{v}^{*}} C_{\alpha}^{\beta \gamma} \frac{\partial S}{\partial v^{\beta}} \frac{\partial S}{\partial v^{\gamma}} d v^{\alpha}
$$

where $S$ defined in (2.7) is now written in the flat coordinates. We use the resonance relations to satisfy the MLG selection rules. Explicitly, the resonance transformation is

$$
\begin{aligned}
t_{m n}= & \lambda_{m n}+\sum_{m_{1}, n_{1}}^{\delta_{m_{1} n_{1}} \leq \delta_{m n}} A_{m n}^{m_{1} n_{1}} \mu^{\delta_{m n}-\delta_{m_{1} n_{1}}} \lambda_{m_{1} n_{1}} \\
& +\sum_{m_{1}, n_{1}, m_{2}, n_{2}}^{\delta_{m_{1} n_{1}}+\delta_{m_{2} n_{2}} \leq \delta_{m n}} A_{m n}^{m_{1} n_{1}, m_{2} n_{2}} \mu^{\delta_{m n}-\delta_{m_{1} n_{1}}-\delta_{m_{2} n_{2}}} \lambda_{m_{1} n_{1}} \lambda_{m_{2} n_{2}}+\ldots,
\end{aligned}
$$

where $\lambda_{m n}$ are the Liouville coupling constants and the constants $A_{m n}^{m_{1} n_{1}}, A_{m n}^{m_{1} n_{1}, m_{2} n_{2}}, \ldots$ are to be defined from the conformal and fusion selection rules. The summation regions in (3.2) are constraint by the following resonance requirement

$$
\sum_{i, j} \delta_{m_{i} n_{j}}+N=\delta_{m n}
$$

where $N$ is some positive integer. The gravitational dimensions $\delta_{m n}$ for the unitary minimal model $M(q+1, q)$ are given by

$$
\delta_{m n}=\frac{2 q+1-|(q+1) m-q n|}{2 q} .
$$

In particular, $\lambda_{11}=\mu$ is the cosmological constant, and $\lambda_{m n} \sim \mu^{\delta_{m n}}$. In what follows, $\lambda_{m n}$ is used only for $(m, n) \neq(1,1)$. For the unitary minimal models, using the reflection symmetry of the Kac table, we can choose the non-equivalent representatives of the primary 
fields in such a way that $1 \leq n \leq m \leq q-1$. In what follows we assume that all the pairs $(m, n)$ and $\left(m_{i}, n_{i}\right)$ satisfy this condition.

By regrouping the terms, we can write the expansion of the action

$$
S=S^{(0)}+\sum_{m, n} \lambda_{m n} S^{(m n)}+\sum_{m_{1}, n_{1}, m_{2}, n_{2}} \lambda_{m_{1} n_{1}} \lambda_{m_{2} n_{2}} S^{\left(m_{1} n_{1}, m_{2} n_{2}\right)}+\ldots
$$

The correlation numbers in the Liouville frame are related to the coefficients in the coupling constant decomposition of the partition function

$$
\mathcal{F}[t(\lambda)]=Z_{0}+\sum_{m_{1}, n_{1}} \lambda_{m_{1} n_{1}} Z_{m_{1} n_{1}}+\sum_{m_{1}, n_{1}} \sum_{m_{2}, n_{2}} \lambda_{m_{1} n_{1}} \lambda_{m_{2} n_{2}} Z_{m_{1} n_{1}, m_{2} n_{2}}+\ldots
$$

In other words, the correlation numbers are expressed in the form

$$
\left\langle O_{m_{1} n_{1}} \ldots O_{m_{N} n_{N}}\right\rangle=Z_{m_{1} n_{1}, \ldots, m_{N} n_{N}}=\left.\frac{\partial}{\partial \lambda_{m_{1} n_{1}}} \cdots \frac{\partial}{\partial \lambda_{m_{N} n_{N}}}\right|_{\lambda=0} \mathcal{F}[t(\lambda)] .
$$

One comment concerning dimensional analysis is in order. It is convenient to use their dimensionless counterparts instead of considering the dimensional quantities. This is achieved by changing variables $v_{1} \rightarrow v_{1} / v_{1}^{*}$. In what follows, we let the same letter denote the new dimensionless variable. Omitted dimensional factors have the form of some powers of $v_{* 1}$. They can be easily reconstructed using the homogeneity of the polynomial $Q$. These factors, of course, do not contribute to the real physical quantities, which are independent of the normalization.

\subsection{One-point functions}

In what follows, our basic tool is the recurrence relation [13]

$$
\frac{\partial^{2} \theta_{\lambda, k+1}}{\partial v_{\alpha} \partial v_{\beta}}=C_{\gamma}^{\alpha \beta} \frac{\partial \theta_{\lambda, k}}{\partial v_{\gamma}}
$$

The consideration is based on the following statement. On the line $v_{i>1}=0$,

$$
\begin{cases}k \text { even }: & \frac{\partial \theta_{\lambda, k}}{\partial v_{\alpha}}=\delta_{\lambda, \alpha} x_{\lambda, k}\left(-\frac{v_{1}}{q}\right)^{\frac{k}{2} q} \\ k \text { odd }: & \frac{\partial \theta_{\lambda, k}}{\partial v_{\alpha}}=\delta_{\lambda, q-\alpha} y_{\lambda, k}\left(-\frac{v_{1}}{q}\right)^{\frac{k-1}{2} q+\lambda}\end{cases}
$$

where

$$
x_{\lambda, k}=\frac{1}{\left(\frac{\lambda}{q}\right)_{\frac{k}{2}}\left(\frac{k}{2}\right) !} \quad \text { and } \quad y_{\lambda, k}=-\frac{1}{\left(\frac{\lambda}{q}\right)_{\frac{k+1}{2}}\left(\frac{k-1}{2}\right) !} .
$$

The proof is given in appendix B.

The first requirement to be satisfied is the zero expectation values of physical operators (except the unity operator). For the $\mathbf{v}^{*}$ solution, we can take the contour of the integration 
along the $v_{1}$ axis. Keeping in mind the comment at the end of the preceding section, we write the one-point function in the form

$$
\left\langle O_{m n}\right\rangle=\int_{0}^{1} C_{q-1}^{\beta \gamma} \frac{\partial S^{(0)}}{\partial v^{\beta}} \frac{\partial S^{(m n)}}{\partial v^{\gamma}} d v_{1} .
$$

Because of the string equation, the derivative in (3.11) acts only on the integrand leading to $S^{(m n)}$, which is the first term of the series expansion of the action (3.5) in the coupling constants

$$
S^{(m n)}=\operatorname{res}_{y=\infty} Q^{\frac{(q+1) m-q n}{q}}+\sum_{m_{1}, n_{1}}^{\delta_{m_{1} n_{1}}<\delta_{m n}} A_{m_{1} n_{1}}^{m n} \mu^{\delta_{m n}-\delta_{m_{1} n_{1}}} \operatorname{res}_{y=\infty} Q^{\frac{(q+1) m_{1}-q n_{1}}{q}}
$$

where $A_{m_{1} n_{1}}^{m n}$ are the coefficients in the resonance relations of the coupling constants. We note that taking into account (3.3) and (3.4), in (3.12) we are left with the pairs $\left(m_{1}, n_{1}\right)$ such that $m_{1}=m$ and $n_{1}<n$.

Using (2.24), we find

$$
S^{(0)}=\underset{y=\infty}{\operatorname{res}}\left[Q^{\frac{2 q+1}{q}}+\mu Q^{\frac{1}{q}}\right]=-\frac{\theta_{1,2}}{c_{1,2}}-\mu \frac{\theta_{1,0}}{c_{1,0}} .
$$

Taking (3.9) into account, we find that $\frac{\partial S^{(0)}}{\partial v^{\beta}}=0$ for $\beta \neq q-1$. Hence, using the properties of the flat coordinates (3.9) we easily confirm our conjecture formulated in the previous section that there exist some special solution of the string equation which is equal to $\mathbf{v}^{*}=\left\{v_{1}^{*}, 0,0, \ldots\right\}$ in the zeroth order in coupling constants. Explicitly, we find

$$
\frac{\partial S^{(0)}}{\partial v_{\alpha}}\left(\mathbf{v}^{*}\right)=0 \quad \text { for } \quad \alpha \neq 1
$$

Hence, the string equation is reduced to the constaint $\frac{\partial S^{(0)}}{\partial v_{1}}\left(\mathbf{v}^{*}\right)=0$, from which the value of the coordinate $v_{1}^{*}$ can be found (see (3.18), (3.19) below).

For the one-point correlation function we get

$$
\left\langle O_{m n}\right\rangle=\int_{0}^{1} C_{q-1}^{q-1, \gamma} \frac{\partial S^{(0)}}{\partial v^{q-1}} \frac{\partial S^{(m n)}}{\partial v^{\gamma}} d v_{1}=\int_{0}^{1} \frac{\partial S^{(0)}}{\partial v_{1}} \frac{\partial S^{(m n)}}{\partial v_{1}} d v_{1}
$$

where we use $C_{\alpha}^{q-1, \beta}=\delta_{\alpha \beta}$ and lower the indices.

Now, the question is for which pairs $(m, n)$ the factors $\frac{\partial S^{(m n)}}{\partial v_{1}}$ are nonzero. Using (3.9) and (3.12), we find that for $m-n=2 k+1$ with integer $k$, the corresponding gravitational dimension of the one-point function $\left\langle O_{m n}\right\rangle$ is

$$
\left[\left\langle O_{m n}\right\rangle\right]=\frac{2 q+1}{q}-\delta_{m n}=(k+1)
$$

i.e., is analytic in $\mu$ and should not be considered [13]. The second option is $m-n$ even and $m=1$. Because $n \leq m$, the only possible pair is $(1,1)$, which corresponds to the unity operator. 
It is instructive to obtain an explicit answer for the partition function

$$
Z_{0}=\frac{1}{2} \int_{0}^{1} d v_{1}\left(\frac{\partial S^{(0)}}{\partial v_{1}}\right)^{2}
$$

where

$$
\frac{\partial S^{(0)}}{\partial v_{1}}=-\frac{1}{c_{1,2}} \frac{\partial \theta_{1,2}}{\partial v_{1}}-\frac{\mu}{c_{1,0}}
$$

The partial derivative $\frac{\partial \theta_{1,2}}{\partial v_{1}}$ can be easily found from (3.8):

$$
\frac{\partial \theta_{1,2}}{\partial v_{1}}=\left(-\frac{1}{q}\right)^{q-2} \frac{v_{1}^{q}}{q} .
$$

From the string equation $\left.\frac{\partial S^{(0)}}{\partial v_{1}}\right|_{v_{1}=v_{1}^{*}}=0$, we find that the cosmological constant, measured in $\left(v_{1}^{*}\right)^{q}$, is

$$
\mu=-q\left(-\frac{1}{q}\right)^{q} \frac{c_{1,0}}{c_{1,2}}
$$

The integral (3.17) can be evaluated explicitly:

$$
Z_{0}=\frac{\left(-\frac{1}{q}\right)^{2 q-4}}{c_{1,2}^{2}} \frac{1}{(1+q)(1+2 q)}
$$

We conclude that nonzero one-point functions appear only for the unity operator and for other operators having nonsingular gravitational dimensions. All singular one-point functions are automatically zero. We also note that when the flat variables are used on the level of one-point functions, the first order coefficients in the resonance expansion remain undetermined because the requirement of the absence of one-point expectation values is satisfied automatically. This is a specific property of the flat coordinate system. Hence, the explicit form of the resonance relation should be fixed from the restrictions arising on the levels of higher-point correlators.

\subsection{Two-point functions}

At the two-point level, the derivatives again act only on the integrand. Therefore, the result is

$$
\left\langle O_{m_{1} n_{1}} O_{m_{2} n_{2}}\right\rangle=\int_{0}^{1} d v_{1} C_{q-1}^{\alpha \beta} \frac{\partial S^{\left(m_{1} n_{1}\right)}}{\partial v^{\alpha}} \frac{\partial S^{\left(m_{2} n_{2}\right)}}{\partial v^{\beta}}+\int_{0}^{1} d v_{1} C_{q-1}^{\alpha \beta} \frac{\partial S^{(0)}}{\partial v^{\alpha}} \frac{\partial S^{\left(m_{1} n_{1}, m_{2} n_{2}\right)}}{\partial v^{\beta}} .
$$

It follows from the same arguments as in the one-point case that the second integral vanishes. Indeed, the second term in (3.22) has the same form as (3.15). So the analysis is again reduced to the analysis of the contributions of the terms like $\underset{y=\infty}{\operatorname{res}} Q^{\frac{(q+1) m_{j}-q n_{j}}{q}}$, such that $\delta_{m_{1} n_{1}}+\delta_{m_{2} n_{2}}=\delta_{m_{j} n_{j}}+N$. We have seen in the previous section that if not 
analytic such contribution can be non-zero only for $m_{j}=n_{j}=1$, which corresponds to the non-universal two-point function. Hence,

$$
\left\langle O_{m_{1} n_{1}} O_{m_{2} n_{2}}\right\rangle=\int_{0}^{1} d v_{1} C_{q-1}^{\alpha \beta} \frac{\partial S^{\left(m_{1} n_{1}\right)}}{\partial v^{\alpha}} \frac{\partial S^{\left(m_{2} n_{2}\right)}}{\partial v^{\beta}},
$$

which because of (2.31) can be written as

$$
\left\langle O_{m_{1} n_{1}} O_{m_{2} n_{2}}\right\rangle=\sum_{\gamma=1}^{q-1}(-q)^{1-\gamma} \int_{0}^{1} d v_{1} v_{1}^{\gamma-1} \frac{\partial S^{\left(m_{1} n_{1}\right)}}{\partial v_{\gamma}} \frac{\partial S^{\left(m_{2} n_{2}\right)}}{\partial v_{\gamma}} .
$$

Our next step is to derive the general form of the partial derivatives $\frac{\partial S^{(m n)}}{\partial v_{\gamma}}$. We find the following consequence of (3.9) for $\frac{\partial S^{(m n)}}{\partial v_{\alpha}}$ :

$$
\begin{cases}(m-n) \text { even }: & \frac{\partial S^{(m n)}}{\partial v_{\alpha}} \sim \delta_{m, \alpha}\left(v_{1}^{\frac{m-n}{2} q}+\ldots\right), \\ (m-n) \text { odd }: & \frac{\partial S^{(m n)}}{\partial v_{\alpha}} \sim \delta_{m, q-\alpha}\left(v_{1}^{\frac{m-n-1}{2} q+m}+\ldots\right),\end{cases}
$$

where dots denote terms involving the cosmological constant $\mu$. We now consider the two point functions (3.24). It follows from the dimensional analysis that

$$
\left[\left\langle O_{m_{1} n_{1}} O_{m_{2} n_{2}}\right\rangle\right]=\frac{2 q+1}{q}-\delta_{m_{1} n_{1}}-\delta_{m_{2} n_{2}}=\frac{m_{1}-n_{1}}{2}+\frac{m_{2}-n_{2}}{2}+\frac{m_{1}+m_{2}}{2 q} .
$$

According to (3.24) and (3.25), there are three different cases. In the case where one of the fields is even while another is odd (for example, $m_{1}-n_{1}$ is even and $m_{2}-n_{2}$ is odd), we find that $m_{1}+m_{2}=q$, and the two-point function in this case is hence analytic in $\mu$ and is disregarded as nonuniversal.

If both differences $m_{1}-n_{1}$ and $m_{2}-n_{2}$ have the same parity, the dimensional analysis shows that

$$
\left\langle O_{m_{1} n_{1}} O_{m_{2} n_{2}}\right\rangle \sim \delta_{m_{1}, m_{2}} \mu^{\frac{m_{1}-n_{1}}{2}+\frac{m_{2}-n_{2}}{2}+\frac{m_{1}}{q}} .
$$

Taking $m_{1} \in[1, q-1]$ into account, we see that the two-point function cannot be analytic in $\mu$ and must therefore be equal to zero once the pairs $\left(m_{1}, n_{1}\right)$ and $\left(m_{2}, n_{2}\right)$ are different. It proves useful to introduce the new variable

$$
t=2\left(\frac{v_{1}}{v_{1}^{*}}\right)^{q}-1
$$

In the case where both fields are even, the degree of the polynomial $\frac{\partial S^{(m n)}}{\partial v_{m}}$ is

$$
\frac{\partial S^{(m n)}}{\partial v_{m}}(t) \sim t^{\frac{m-n}{2}}+\ldots,
$$

where the dots denote terms subleading in $t$. The diagonality condition for the two-point correlation function in terms of the new variable takes the form

$$
\left\langle O_{m_{1} n_{1}} O_{m_{2} n_{2}}\right\rangle \sim \delta_{m_{1}, m_{2}} \int_{-1}^{1} d t(1+t)^{\frac{m_{1}+m_{2}}{2 q}-1} \frac{\partial S^{\left(m_{1} n_{1}\right)}}{\partial v_{m_{1}}} \frac{\partial S^{\left(m_{2} n_{2}\right)}}{\partial v_{m_{2}}}=0 .
$$


In the case where both fields are odd, it is convenient to change $\frac{\partial S^{(m n)}}{\partial v_{\alpha}}=(1+t)^{\frac{m}{q}} \frac{\partial \tilde{S}^{(m n)}}{\partial v_{\alpha}}$. The degree of the new polynomial is

$$
\frac{\partial \tilde{S}^{(m n)}}{\partial v_{\alpha}} \sim t^{\frac{m-n-1}{2}}+\ldots
$$

and the diagonality condition is

$$
\left\langle O_{m_{1} n_{1}} O_{m_{2} n_{2}}\right\rangle \sim \delta_{m_{1}, m_{2}} \int_{-1}^{1} d t(1+t)^{\frac{m_{1}+m_{2}}{2 q}} \frac{\partial \tilde{S}^{\left(m_{1} n_{1}\right)}}{\partial v_{q-m_{1}}} \frac{\partial \tilde{S}^{\left(m_{2} n_{2}\right)}}{\partial v_{q-m_{2}}}=0 .
$$

Equations (3.30) and (3.32) show that the diagonal form of the two-point correlation numbers requires that the polynomials (3.29) and (3.31) form an orthogonal set of polynomials. Such polynomials are known as Jacobi orthogonal polynomials. In our case we are dealing with the special class of the Jacobi polynomials $P_{n}^{(0, b)}(t)$. They are $n$th order polynomials that form an orthogonal system on the interval $[0,1]$ with the orthogonality condition

$$
\int_{-1}^{1} d t(1+t)^{b} P_{n}^{(0, b)}(t) P_{m}^{(0, b)}(t)=\frac{2^{b+1}}{2 n+b+1} \delta_{m, n}
$$

In the standard normalization, $P_{n}^{(0, b)}(1)=1$. We note that the Jacobi polynomials in this normalization have the highest coefficient

$$
P_{n}^{(0, b)}(t)=\frac{(b+n+1)_{n}}{n !}\left(\frac{t}{2}\right)^{n}+\ldots
$$

Summarizing these results, we conclude that

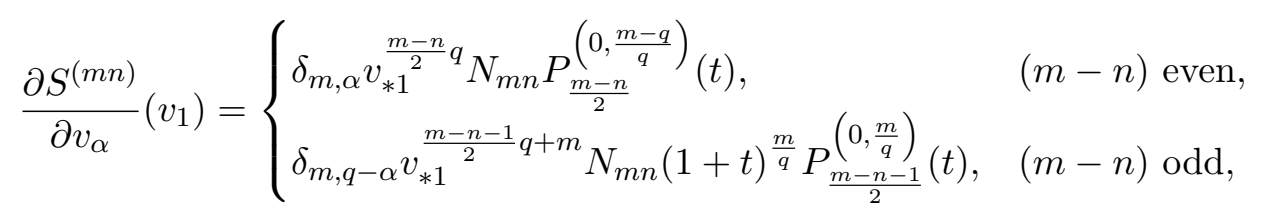

where $t$ is defined in (3.28) and $N_{m n}$ is the normalization factor independent of $t$. Although we obtained (3.35) for $v_{i>1}=0$, the general form of $S^{(m n)}$ can be easily reconstructed using equation (3.12). Hence, we have the answer for the action $S$ defined in (3.5) up to second order in the coupling constant expansion. This result is required for calculating multipoint correlation functions.

\section{Conclusions}

In this paper, we presented the solution of the Douglas string equation and the explicit form of the resonance relations up to second order in the coupling constant expansion, which lead to satisfying the main requirements of minimal Liouville gravity, namely, the requirements that the one-point correlation function except the unity operator must be zero and that the two-point correlators must be diagonal. Using the connection between the Douglas approach and the Frobenius manifold structure, we showed that the appropriate solution of the string equation has a remarkably simple form in the flat coordinates. Calculations 
of the multipoint correlation functions require this information. In addition, it requires knowing the structure constants of the Frobenius algebra. We found the structure constants in the canonical coordinates as well as the expression for the structure constants on the solution of the string equation in the flat coordinates, i.e., for all except the first coordinates be zero. To obtain the general answer for the partition function, which is appropriate for calculating arbitrary multipoint correlators, the explicit form of the general structure constants in the flat coordinates is required. The general form of the resonance relations is also needed. Presumably, higher terms in the coupling constant decomposition are fixed from the restrictions following from the fusion rules. We are going to study this question in the near future.

\section{Acknowledgments}

I am grateful to A. Belavin for useful comments and discussions and K. Alkalaev for comments on the draft of this article. I thank G. Mussardo, SISSA and K. Narain, ICTP, Trieste, Italy for the hospitality during my visits in 2013. The research was performed under a grant funded by Russian Science Foundation (project No. 14-12-01383).

\section{A $C_{\alpha \beta \gamma}$}

If two of the indices are raised, then the structure constants in $\mathbf{u}$ coordinates is known from (2.17). On the other hand, the metric in flat coordinates is simple, and lowering an index $\alpha$ hence is just replacing it with $q-\alpha$. Therefore,

$$
C_{\alpha \beta \gamma}=\frac{\partial v^{q-\alpha}}{\partial u^{i}} \frac{\partial v^{q-\beta}}{\partial u^{j}} \frac{\partial u^{k}}{\partial v^{\gamma}} \widetilde{C}_{k}^{i j}
$$

Using the explicit form of the coordinate transformation (2.21), we find that on the line $v_{k>1}=0$, we have

$$
\begin{aligned}
& \frac{\partial u^{k}}{\partial v^{\gamma}}(\mathbf{v})=U_{\gamma}^{k} \\
& \frac{\partial v^{\alpha}}{\partial u^{i}}(\mathbf{v})=V_{\gamma}^{k}
\end{aligned}
$$

where

$$
\begin{aligned}
& U_{\gamma}^{k}=\left(\begin{array}{c}
\frac{\gamma-k+q-2}{2} \\
\frac{\gamma+k-q}{2}
\end{array}\right)\left(\frac{v_{1}}{2}\right)^{\frac{\gamma+k-q}{2}} \quad \text { if } \quad \frac{\gamma+k-q}{2} \in \mathbb{N}, \quad \text { otherwise } 0, \\
& V_{j}^{\rho}=\frac{2 \rho}{q+\rho-j}\left(\begin{array}{c}
q-j-1 \\
\frac{q-\rho-j}{2}
\end{array}\right)\left(-\frac{v_{1}}{2}\right)^{\frac{q-\rho-j}{2}} \quad \text { if } \quad \frac{q-\rho-j}{2} \in \mathbb{N}, \quad \text { otherwise } 0 .
\end{aligned}
$$

In particular, from (A.4), we find

$$
u^{k}=\frac{2 q}{k+1}\left(\begin{array}{c}
\frac{2 q-k-1}{2} \\
\frac{k-1}{2}
\end{array}\right)\left(\frac{v_{1}}{2}\right)^{\frac{k+1}{2}} \text { if } \quad \frac{k+1}{2} \in \mathbb{N}, \quad \text { otherwise } 0 .
$$

In this notation, we find

$$
C_{\alpha \beta \gamma}\left(\mathbf{v}_{*}\right)=V_{j}^{q-\alpha} V_{j}^{q-\beta} U_{\gamma}^{k} \widetilde{C}_{k}^{i j} .
$$

Using standard identities for the binomial coefficients, we obtain (2.31). 


\section{B Evaluation of $\frac{\partial \theta_{\lambda, k}}{\partial v_{\alpha}}$}

We use our basic recurrence relation

$$
\frac{\partial^{2} \theta_{\lambda, k}}{\partial v_{1} \partial v_{\alpha}}=C_{\gamma}^{1 \alpha} \frac{\partial \theta_{\lambda, k-1}}{\partial v_{\gamma}}
$$

We integrate this equation over $v_{1}$ to find

$$
\frac{\partial \theta_{\lambda, k}}{\partial v_{\alpha}}=\int^{v_{1}} C_{\gamma}^{1 \alpha} \frac{\partial \theta_{\lambda, k-1}}{\partial v_{\gamma}} d v_{1}
$$

where the integration constant is absent because it is independent of $v_{1}$ and can hence depend on only $v_{\alpha>1}$, which are set to zero. Using this equation iteratively, we find

$$
\frac{\partial \theta_{\lambda, k}}{\partial v_{\alpha}}=\int^{v_{1}} d v_{1}^{(1)} \ldots \int^{v_{1}^{(k-1)}} d v_{1}^{(k)} C_{\gamma_{1}}^{1 \alpha}\left(v_{1}^{(1)}\right) C_{\gamma_{2}}^{1 \gamma_{1}}\left(v_{1}^{(2)}\right) \ldots C_{\gamma_{k}}^{1 \gamma_{k-1}}\left(v_{1}^{(k)}\right) \frac{\partial \theta_{\lambda, 0}}{\partial v_{\gamma_{k}}}
$$

where explicitly $\frac{\partial \theta_{\lambda, 0}}{\partial v_{\gamma_{k}}}=\delta_{\lambda, \gamma_{k}}$. From the explicit form of the structure constant (2.31), we conclude that

$$
\begin{aligned}
& \gamma_{1}=\gamma_{3}=\cdots=q-\alpha \\
& \gamma_{2}=\gamma_{4}=\cdots=\alpha .
\end{aligned}
$$

In particular, we find that $\lambda=\alpha$ for even $k$ while $\lambda=q-\alpha$ for odd $k$. Otherwise, the integral is equal to zero. After contracting all the indices, we are left with a multiple integral containing

$$
C_{q-\alpha}^{1 \alpha}\left(v_{1}^{(i)}\right)=\left(-\frac{v_{1}^{(i)}}{q}\right)^{q-\alpha-1}
$$

and

$$
C_{\alpha}^{1 q-\alpha}\left(v_{1}^{(i)}\right)=\left(-\frac{v_{1}^{(i)}}{q}\right)^{\alpha-1}
$$

Explicit integration gives

$$
\left\{\begin{array}{l}
k \text { even }: \quad \frac{\partial \theta_{\lambda, k}}{\partial v_{\alpha}}=\delta_{\lambda, \alpha}\left(-\frac{1}{q}\right)^{\frac{k}{2}(q-2)} \frac{v_{1}^{\frac{k}{2} q}}{\alpha q(\alpha+q) 2 q(\alpha+2 q) 3 q \ldots\left(\alpha+\left(\frac{k}{2}-1\right) q\right)\left(\frac{k}{2} q\right)}, \\
k \text { odd }: \quad \frac{\partial \theta_{\lambda, k}}{\partial v_{\alpha}}=\delta_{\lambda, q-\alpha}\left(-\frac{1}{q}\right)^{\frac{k-1}{2}(q-2)+q-\alpha-1} \frac{v_{1}^{2} q-\alpha}{(q-\alpha) q(2 q-\alpha) 2 q(3 q-\alpha) 3 q \ldots\left(\frac{k-1}{2} q\right)\left(\frac{k+1}{2} q-\alpha\right)} .
\end{array}\right.
$$

This gives (3.9) and (3.10).

Open Access. This article is distributed under the terms of the Creative Commons Attribution License (CC-BY 4.0), which permits any use, distribution and reproduction in any medium, provided the original author(s) and source are credited. 


\section{References}

[1] A.M. Polyakov, Quantum Geometry of Bosonic Strings, Phys. Lett. B 103 (1981) 207 [INSPIRE].

[2] A.A. Belavin, A.M. Polyakov and A.B. Zamolodchikov, Infinite Conformal Symmetry in Two-Dimensional Quantum Field Theory, Nucl. Phys. B 241 (1984) 333 [inSPIRE].

[3] V.A. Kazakov, A.A. Migdal and I.K. Kostov, Critical Properties of Randomly Triangulated Planar Random Surfaces, Phys. Lett. B 157 (1985) 295 [InSPIRE].

[4] V.A. Kazakov, Ising model on a dynamical planar random lattice: Exact solution, Phys. Lett. A 119 (1986) 140 [INSPIRE].

[5] V.A. Kazakov, The Appearance of Matter Fields from Quantum Fluctuations of $2 D$ Gravity, Mod. Phys. Lett. A 4 (1989) 2125 [INSPIRE].

[6] M. Staudacher, The Yang-lee Edge Singularity on a Dynamical Planar Random Surface, Nucl. Phys. B 336 (1990) 349 [inSPIRE].

[7] E. Brézin and V.A. Kazakov, Exactly Solvable Field Theories of Closed Strings, Phys. Lett. B 236 (1990) 144 [INSPIRE].

[8] M.R. Douglas and S.H. Shenker, Strings in Less Than One-Dimension, Nucl. Phys. B 335 (1990) 635 [INSPIRE].

[9] D.J. Gross and A.A. Migdal, Nonperturbative Two-Dimensional Quantum Gravity, Phys. Rev. Lett. 64 (1990) 127 [INSPIRE].

[10] V.G. Knizhnik, A.M. Polyakov and A.B. Zamolodchikov, Fractal Structure of $2 D$ Quantum Gravity, Mod. Phys. Lett. A 3 (1988) 819 [inSPIRE].

[11] G.W. Moore, N. Seiberg and M. Staudacher, From loops to states in 2-D quantum gravity, Nucl. Phys. B 362 (1991) 665 [inSPIRE].

[12] A.A. Belavin and A.B. Zamolodchikov, On Correlation Numbers in 2D Minimal Gravity and Matrix Models, J. Phys. A 42 (2009) 304004 [arXiv:0811.0450] [INSPIRE].

[13] A. Belavin, B. Dubrovin and B. Mukhametzhanov, Minimal Liouville Gravity correlation numbers from Douglas string equation, JHEP 01 (2014) 156 [arXiv:1310.5659] [INSPIRE].

[14] M.R. Douglas, Strings in Less Than One-dimension and the Generalized $K^{-} D^{-} V$ Hierarchies, Phys. Lett. B 238 (1990) 176 [INSPIRE].

[15] P.H. Ginsparg, M. Goulian, M.R. Plesser and J. Zinn-Justin, $(p, q)$ string actions, Nucl. Phys. B 342 (1990) 539 [INSPIRE].

[16] B. Dubrovin, Integrable systems in topological field theory, Nucl. Phys. B 379 (1992) 627 [INSPIRE].

[17] P. Di Francesco and D. Kutasov, Unitary minimal models coupled to 2-D quantum gravity, Nucl. Phys. B 342 (1990) 589 [inSPIRE]. 